\title{
Optimum Accelerated Overrelaxation Method in a Special Case
}

\author{
By G. Avdelas and A. Hadjidimos
}

\begin{abstract}
In this paper we give the optimum parameters for the Accelerated Overrelaxation (AOR) method in the special case where the matrix coefficient of the linear system, which is solved, is consistently ordered with nonvanishing diagonal elements. Under certain assumptions, concerning the eigenvalues of the corresponding Jacobi matrix, it is shown that the optimum AOR method gives better convergence rates than the optimum SOR does, while in the remaining cases the optimum AOR method coincides with the optimum SOR one.
\end{abstract}

1. Introduction. For the numerical solution of the linear system

$$
A x=b,
$$

where $A$ is a nonsingular matrix with nonvanishing diagonal elements of order $N, x$ and $b N$-dimensional vectors with $x$ unknown and $b$ known, a linear stationary two-parameter iterative scheme, called Accelerated Overrelaxation (AOR) method, was introduced in [1] by the second of the present authors. Assuming, without loss of generality, that $A$ can be split as follows

$$
A=I-L-U
$$

where $I$ is the unit matrix of order $N$ and $-L$ and $-U$ are the strictly lower and upper triangular parts of $A$, respectively, the AOR scheme of [1] has the form

$$
\begin{array}{r}
x^{(n+1)}=(I-\omega L)^{-1}[(1-r) I+(r-\omega) L+r U] x^{(n)}+r(I-\omega L)^{-1} b, \\
n=0,1,2, \ldots .
\end{array}
$$

In (1.2) $x^{(0)}$ is any arbitrary $N$-dimensional vector and $r \neq 0$ and $\omega$ are two parameters called acceleration and overrelaxation factors, respectively. As was noticed in [1], when $(r, \omega)=(1,0),(1,1),(r, 0)$ and $(\omega, \omega),(1.2)$ reduces to the Jacobi, Gauss-Seidel, Simultaneous Overrelaxation and Successive Overrelaxation methods, respectively; see e.g. [2], [3] and [4]. It was also noticed there that for $\omega \neq 0$ the AOR method is an extrapolation of the SOR method with relaxation factor $\omega$ and extrapolation factor $r / \omega$. In the special case where $A$ is a consistently ordered matrix, a relationship between the eigenvalues $\lambda$ of the AOR iterative matrix, given by

$$
L_{r, \omega}=(I-\omega L)^{-1}[(1-r) I+(r-\omega) L+r U],
$$

and the eigenvalues $\mu$ of the corresponding Jacobi matrix $L_{1,0}$ was established in [1]. This is the following

$$
\lambda^{2}-\left[2(1-r)+r \omega \mu^{2}\right] \lambda+(r-1)^{2}+(\omega-r) r \mu^{2}=0
$$

Received June 30, 1978; revised April 28, 1980.

1980 Mathematics Subject Classification. Primary 65F10. 
It is the purpose of this paper to give the optimum parameters (and therefore the optimum AOR method) in this special case in such a way that the spectral radius of (1.3) is minimized. This is what we do in the next section under the additional assumptions that the eigenvalues of $L_{1,0}$ are all real and less than 1 in modulus, as, for example, in the case where $A$ is symmetric and positive definite.

2. Determination of the Optimum Parameters. Let $\mu_{i} \mid i=1(1) N$ be the real eigenvalues of $L_{1,0}$, which are less than one in modulus. We define

$$
0 \leqslant \underline{\mu} \equiv \min _{i}\left|\mu_{i}\right| \leqslant \max _{i}\left|\mu_{i}\right| \equiv \bar{\mu}<1 \text {. }
$$

If now $\lambda_{1}$ and $\lambda_{2}$ are the two roots of (1.4) in terms of $\mu \in[\mu, \bar{\mu}], r \in(-\infty,+\infty)$ and $\omega \in(-\infty,+\infty)$, then the problem of minimizing the spectral radius (1.3) is that of determining the pair $(r, \omega)$ which minimizes the following expression

$$
\min _{r}\left\{\max _{\mu}\left\{\max \left\{\left|\lambda_{1}\right|,\left|\lambda_{2}\right|\right\}\right\}\right\}
$$

with respect to (wrt) $\omega$. In (2.1) we do not impose any extra restrictions on the parameters in order to have it less than 1 , since we already know that for $r=\omega=2 /\left(1+\left(1-\bar{\mu}^{2}\right)^{1 / 2}\right)$ (i.e. in the optimum SOR method) the corresponding spectral radius is less than 1 . Therefore, in what follows, our objective will be that of determining local minima for the expression (2.1), which may be less (better) than the expression $\left(1-\left(1-\bar{\mu}^{2}\right)^{1 / 2}\right) /\left(1+\left(1-\bar{\mu}^{2}\right)^{1 / 2}\right)$, giving the spectral radius of the optimum SOR method, and among them, if there are any, to select the smallest one. It is noted that in the detailed analysis, which is rather elementary but very long and cumbersome and is not to be given here, the following basic cases are treated separately:

$$
\begin{array}{ll}
\text { (i) } & 0=\underline{\mu}, \\
\text { (ii) } & 0<\underline{\mu}<\bar{\mu}, \\
& \text { (iia) }\left(1-\bar{\mu}^{2}\right)^{1 / 2} \leqslant 1-\underline{\mu}^{2}, \\
& \text { (iib) } 1-\underline{\mu}^{2}<\left(1-\bar{\mu}^{2}\right)^{1 / 2}, \\
\text { (iii) } & 0<\underline{\mu}=\bar{\mu} .
\end{array}
$$

In each one of the cases (2.2), we consider various intervals from which the parameter $\omega$ can take its values, and for each one of these we consider other intervals associated with the parameter $r$. Thus, we have to examine a tremendous number of cases and this is the reason why we cannot present the detailed analysis here. However, for the interested reader we give, in what follows, the essential elements on which the aforementioned analysis is based.

First, we consider the expression $D(\mu) \equiv \mu^{2} \omega^{2}-4 \omega+4$, which is one of the factors of the discriminant of (1.4), the other being $r^{2} \mu^{2}$, and plays a very important role. We have therefore to distinguish three basic cases:

A: $D(\bar{\mu}) \leqslant 0$. This implies $D(\mu) \leqslant 0$ for any $\mu \in[\underline{\mu}, \bar{\mu}]$, so that the roots of (1.4) are complex conjugate ones. Hence we shall have

$$
E \equiv E_{1}(r, \omega, \mu) \equiv \max \left\{\left|\lambda_{1}\right|,\left|\lambda_{2}\right|\right\}=\left(\left(1-\mu^{2}\right) r^{2}-\left(2-\omega \mu^{2}\right) r+1\right)^{1 / 2}
$$


B: $D(\underline{\mu}) \geqslant 0$. It is implied that $D(\mu) \geqslant 0$ for any $\mu \in[\underline{\mu}, \bar{\mu}]$, so that the roots of (1.4) are real. Consequently

$$
E \equiv E_{2}(r, \omega, \mu) \equiv \max \left\{\left|\lambda_{1}\right|,\left|\lambda_{2}\right|\right\}=\left(|H|+|\omega| \mu(D(\mu))^{1 / 2}\right) / 2,
$$

where

$$
H \equiv H(r, \omega, \mu) \equiv 2(1-r)+r \omega \mu^{2} .
$$

C: $D(\mu) \leqslant 0 \leqslant D(\bar{\mu})$. In this case there exists a real number $\mu_{d}=4(\omega-1) / \omega^{2}$ $\in[\mu, \mu]$ such that $D\left(\mu_{d}\right)=0$. Consequently, in order to find the quantity $E$ for $\mu \in\left[\underline{\mu}, \mu_{d}\right]$, (2.3) has to be considered, while for $\mu \in\left[\mu_{d}, \bar{\mu}\right](2.4)$ is to be taken. Thus, we have that

$$
E \equiv \max \left\{\underset{\mu \in\left[\underline{\mu}, \mu_{d}\right]}{E_{1}}(r, \omega, \mu), \underset{\mu \in\left[\mu_{d}, \bar{\mu}\right]}{E_{2}}(r, \omega, \mu)\right\} .
$$

Secondly, we introduce some notations. Thus, the four roots of the two quadratics $D(\bar{\mu})$ and $D(\underline{\mu})$ are denoted in their order of magnitude as in (2.7) below

$$
\begin{aligned}
\tilde{\omega}_{1} & \equiv 2 /\left(1+\left(1-\underline{\mu}^{2}\right)^{1 / 2}\right) \leqslant \tilde{\omega}_{2} \equiv 2 /\left(1+\left(1-\bar{\mu}^{2}\right)^{1 / 2}\right) \\
& <\tilde{\omega}_{3} \equiv 2 /\left(1-\left(1-\bar{\mu}^{2}\right)^{1 / 2}\right) \leqslant \tilde{\omega}_{4} \equiv 2 /\left(1-\left(1-\underline{\mu}^{2}\right)^{1 / 2}\right),
\end{aligned}
$$

where it is noted that for $\underline{\mu}=0, \tilde{\omega}_{1}=1$ and $\tilde{\omega}_{4}=\infty$. Thirdly, we define the quantities

$$
F \equiv \max _{\mu} E, \quad G \equiv \min _{r} F, \quad \rho \equiv \min _{\omega} G,
$$

which are very useful. And, finally, we state and prove three lemmas denoted by $L_{1}, L_{2}, L_{3}$, respectively:

Lemma $1\left(\mathrm{~L}_{1}\right)$. If $D(\bar{\mu}) \leqslant 0$ and $r(\omega-r) \leqslant 0, F$ takes place for $\mu=\underline{\mu}$.

Proof. Since $D(\bar{\mu}) \leqslant 0, E$ is given by the RHS of $(2.3)$, or equivalently by the square root of the constant term of (1.4), it is evident from the last expression and from the fact that $r(\omega-r) \leqslant 0$, that $F=\max _{\mu} E=E_{1}(r, \omega, \underline{\mu})$.

Lemma $2\left(L_{2}\right)$. If $D(\mu) \geqslant 0$, then $\left(\mu^{2} \omega^{2}-2 \omega+2\right) \pm \mu \omega(D(\mu))^{1 / 2}>0$.

Proof. Because of the assumption we observe that

$$
\mu^{2} \omega^{2}-2 \omega+2=\left(\mu^{2} \omega^{2}+D(\mu)\right) / 2>0 .
$$

In order to prove the relationship given it is sufficient and necessary to prove that $\left(\mu^{2} \omega^{2}-2 \omega+2\right)^{2}-\mu^{2} \omega^{2}\left(\mu^{2} \omega^{2}-4 \omega+4\right)=4(\omega-1)^{2}$ is greater than or equal to zero, which is true.

Lemma $3\left(\mathrm{~L}_{3}\right)$. If $D(\bar{\mu}) \geqslant 0$ and $\omega \geqslant 2 / \bar{\mu}^{2}$, then $\left(2-\omega \bar{\mu}^{2}\right) \pm \bar{\mu}(D(\bar{\mu}))^{1 / 2}<0$.

Proof. Because of the second assumption, we have that $2-\omega \bar{\mu}^{2} \leqslant 0$. Hence, in order to prove the relationship given, it is sufficient and necessary to prove that $\left(2-\omega \bar{\mu}^{2}\right)^{2}-\bar{\mu}^{2}\left(\bar{\mu}^{2} \omega^{2}-4 \omega+4\right)=4\left(1-\bar{\mu}^{2}\right) \geqslant 0$, which always holds.

After the detailed analysis takes place, we are able to give, as a summary, in a self-explained table, the optimum results which correspond to the basic cases (2.2). 
TABLE

Optimum Values

\begin{tabular}{|l|c|c|c|c|}
\hline Case & Identification & $\begin{array}{c}\text { Overrelaxation } \\
\text { factor } \omega\end{array}$ & $\begin{array}{c}\text { Acceleration } \\
\text { factor } r\end{array}$ & $\begin{array}{c}\text { Spectral } \\
\text { radius } \rho\end{array}$ \\
\hline (i) & $\underline{\mu}=0$ & $\tilde{\omega}_{2}^{*}$ & $\tilde{\omega}_{2}$ & $\frac{1-\left(1-\bar{\mu}^{2}\right)^{1 / 2}}{1+\left(1-\bar{\mu}^{2}\right)^{1 / 2}}$ \\
\hline (iia) & $\begin{array}{c}0<\underline{\mu}<\bar{\mu} \\
\left(1-\bar{\mu}^{2}\right)^{1 / 2}<1-\underline{\mu}^{2}\end{array}$ & $\tilde{\omega}_{2}$ & $\tilde{\omega}_{2}$ & $\frac{1-\left(1-\bar{\mu}^{2}\right)^{1 / 2}}{1+\left(1-\bar{\mu}^{2}\right)^{1 / 2}}$ \\
\hline (iib) & $\begin{array}{c}0<\mu<\bar{\mu} \\
1-\underline{\mu}^{2}<\left(1-\bar{\mu}^{2}\right)^{1 / 2}\end{array}$ & $\tilde{\omega}_{2}$ & $\frac{1-\underline{\mu}^{2}-\left(1-\bar{\mu}^{2}\right)^{1 / 2}}{\left(1-\underline{\mu}^{2}\right)\left(1+\left(1-\bar{\mu}^{2}\right)^{1 / 2}\right)}$ & $\frac{\underline{\mu}\left(\bar{\mu}^{2}-\underline{\mu}^{2}\right)^{1 / 2}}{\left(1-\underline{\mu}^{2}\right)^{1 / 2}\left(1+\left(1-\bar{\mu}^{2}\right)^{1 / 2}\right)}$ \\
\hline \multirow{2}{*}{ (iii) } & $0<\underline{\mu}=\bar{\mu}$ & $\tilde{\omega}_{2}$ & $1 /\left(1-\bar{\mu}^{2}\right)^{1 / 2}$ & 0 \\
\cline { 2 - 5 } & \multirow{2}{*}{0} & $\tilde{\omega}_{2}$ & $-1 /\left(1-\bar{\mu}^{2}\right)^{1 / 2}$ & 0 \\
\hline
\end{tabular}

$* \tilde{\omega}_{2}$ is given in (2.7).

As is seen from the table of optimum values in the cases (i) and (iia), the optimum AOR method coincides with the optimum SOR one, while, in the cases (iib) and (iii), the optimum AOR is better than the optimum SOR. It should be pointed out that the optimum results of case (iii) were also obtained in [1]; see Theorem 3, Section 5.

3. Numerical Examples. To show that, in the cases (iib) and (iii) of the table, the optimum convergence rates of the AOR method are much better than the corresponding ones of the optimum SOR, we present two numerical examples. In each one of them, the matrix coefficient $A$ of system (1.1) is a consistently ordered matrix of order four and of the form $I-L-U$, so that the associated Jacobi matrices, their corresponding eigenvalues, and all the other optimum parameters involved are readily evaluated.

(i) Let

$$
A=\left[\begin{array}{cccc}
1 & 0 & \frac{1}{5} & \frac{1}{5} \\
0 & 1 & -\frac{71}{10} & \frac{113}{10} \\
\frac{16}{5} & \frac{1}{5} & 1 & 0 \\
2 & \frac{1}{5} & 0 & 1
\end{array}\right]
$$

The eigenvalues of its Jacobi matrix are $\pm \sqrt{23} / 5$ and $\pm 2 \sqrt{6} / 5$ so that $\mu$ $=\sqrt{23} / 5$ and $\bar{\mu}=2 \sqrt{6} / 5<1$. These bounds satisfy the restrictions of case (iib) since $0<\mu=\sqrt{23} / 5<\bar{\mu}=2 \sqrt{6} / 5$ and $1-\mu^{2}=2 / 25<\left(1-\bar{\mu}^{2}\right)^{1 / 2}=1 / 5$. Therefore the optimum values of the table apply. These, for the AOR method, are $(r, \omega)=(-5 / 4,5 / 3)$ and $\rho\left(L_{r, \omega}\right)=\sqrt{46} / 12$, while for the SOR one these are $(\omega, \omega)=(5 / 3,5 / 3)$ and $\rho\left(L_{\omega, \omega}\right)=2 / 3$. It is readily seen that $\rho\left(L_{r, \omega}\right)=\sqrt{46} / 12<$ $\rho\left(L_{\omega, \omega}\right)=2 / 3$. 
(ii) Let

$$
A=\left[\begin{array}{cccc}
1 & 0 & \frac{1}{5} & \frac{1}{5} \\
0 & 1 & -\frac{18}{5} & 6 \\
\frac{24}{5} & \frac{1}{5} & 1 & 0 \\
\frac{12}{5} & \frac{1}{5} & 0 & 1
\end{array}\right] .
$$

This time the eigenvalues of the Jacobi matrix are $\pm 2 \sqrt{6} / 5$, each one with multiplicity two. Thus $0<\mu=2 \sqrt{6} / 5=\bar{\mu}<1$. Since the restrictions of case (iii) are satisfied, the corresponding optimum values of the table apply. The optimum results for the SOR method are the same as before. However, the optimum results for the AOR method are $(r, \omega)=(5,5 / 3)$ and $(r, \omega)=(-5,5 / 2)$, where both pairs give $\rho\left(L_{r, \omega}\right)=0<\rho\left(L_{\omega, \omega}\right)=2 / 3$, that is, a drastic reduction in the convergence rates.

Department of Mathematics

University of Ioannina

Ioannina, Greece

1. A. Hadjidmos, “Accelerated overrelaxation method,” Math. Comp., v. 32, 1978, pp. 149-157.

2. R. S. VARgA, Matrix Iterative Analysis, Prentice-Hall, Englewood Cliffs, N. J., 1962.

3. E. L. WACHSPress, Iterative Solution of Elliptic Systems and Applications to the Neutron Diffusion Equations of Reactor Physics, Prentice-Hall, Englewood Cliffs, N. J., 1966.

4. D. M. Young, Iterative Solution of Large Linear Systems, Academic Press, New York and London, 1971. 